\title{
Effect of Nicotine on Dopamine and Glutathione Levels in Presence of Oligoelements in Brain Regions of Young Rats*
}

\author{
—_Effect of Nicotine on Brain Regions of Rat
}

\author{
David Calderón Guzmán ${ }^{1}$, Ernestina Hernández García ${ }^{1}$, Francisca Trujillo Jiménez², \\ Gerardo Barragán Mejía ${ }^{1}$, Hugo Juárez Olguín ${ }^{2,3 \#, ~ J o s e ́ ~ A . ~ S a l d i v a r ~ G o n z a ́ l e z ~}{ }^{3}$, \\ Daniel Santamaria del Angel ${ }^{1}$, Norma Osnaya Brizuela ${ }^{4}$
}

\begin{abstract}
${ }^{1}$ Laboratorio de Neuroquímica, Instituto Nacional de Pediatría (INP), México City, México; ${ }^{2}$ Laboratorio de Farmacología, INP, México City, México; ${ }^{3}$ Departamento de Farmacología, Facultad de Medicina, Universidad Nacional Autónoma de México, México City, México; ${ }^{4}$ Laboratorio de Patología Experimental, México City, México.
\end{abstract}

Email: \#juarezol@yahoo.com

Received May $25^{\text {th }}, 2012$; revised June $29^{\text {th }}, 2012$; accepted July $5^{\text {th }}, 2012$

\begin{abstract}
Aim: The purpose of this study was to understand the mechanism of nicotine mediated addiction and the role of oligoelements in reducing its effect. Methods: Male Wistar rats (weight $80 \mathrm{~g}$ ) were treated with single and repeated doses of nicotine and/or oligoelements as follows: group 1 (control) $\mathrm{NaCl} 0.9 \%$; group 2, nicotine $(1 \mathrm{mg} / \mathrm{kg}$ ); group 3, oligoelements $(50 \mu \mathrm{l} / \mathrm{rat})$; and group 4 , nicotine $(1 \mathrm{mg} / \mathrm{kg})+$ oligoelements $(50 \mu \mathrm{l} / \mathrm{rat})$. All drugs were intraperitoneally administered for 4 days. Blood for the measurement of glucose was obtained from all the animals. Samples of the brain regions (cortex, hemispheres and cerebellum + medulla oblongata) of each rat were obtained and used to measure the concentrations of dopamine, GSH levels, and lipid peroxidation (TBARS) using fluorescence and spectrophotometric methods. Results: Glucose level increased in rats treated with nicotine and oligoelements $(p<0.05)$, while GSH level decreased in cerebellum/medulla oblongata and hemispheres $(p<0.05)$ of the same animals. TBARS levels increased in cerebellum/medulla oblongata and hemispheres of animals treated with nicotine and oligoelements, but decreased in the same regions $(\mathrm{p}<0.05)$ in rats treated only with oligoelements. The levels of dopamine decreased in cortex and hemispheres, but increased in cerebellum/medulla and oblongata regions of rats treated with both compounds $(\mathrm{p}<0.05)$. Conclusions: Nicotine and oligoelements are associated with increase in the level of glucose, an effect that was more pronounced in the group treated with both drugs. Reduction of oxidative stress and dopamine metabolism may be involved in this effect.
\end{abstract}

Keywords: Glutathione; Lipid Peroxidation; Oxidative Stress

\section{Introduction}

In Mexico, drug addictions in adolescents $(53.5 \%$ in men and $63.2 \%$ in women) begin with tobacco. In our country, smoking usually begins in mid-adolescence ( 15 years old) and smokers between 18 and 65 years old represent $20.6 \%$ of the population [1], a figure which is presently with an increasing tendency. Nicotine is an alkaloid found in tobacco plant (Nicotiana tabacum). In low concentrations, nicotine has stimulating effect and constitutes one of the principal causes of tobacco addiction. Majority of cigarettes in the world contain between 1 and $2 \mathrm{mg}$ or more of nicotine. On smoking, a smoker usually inhales $0.8 \mathrm{mg}$ of nicotine per cigarette [2].

\footnotetext{
"Declaration of interest: We declare that there is no conflict of interest

${ }^{\#}$ Corresponding author.
}

It is known that dopamine is an appetite suppressant, and the administration of nicotine, regardless of the way, acutely increases the levels of neurotransmitters in the brain, thereby reducing the need for energy intake and consequently suppressing the appetite [3], although the mechanisms are unclear.

Nicotine has been documented as a possible contributing factor to the current nutrition-related chronic diseases epidemic [1]. However, recent studies have shown that micronutrient deficiencies are still significant public health problems, affecting the most vulnerable age groups and gender [4]. Likewise, there are some food rich in oligoelements like milk for daily intake [5], to offset this deficiency.

The vital biological processes need oligoelements as Zinc $(\mathrm{Zn})$, Manganese (Mn), Copper $(\mathrm{Cu})$, and others, all 
of which are integral part of many important enzymes. $\mathrm{Cu}$ is essential for life and their excessive accumulation in the brain decreases GSH levels [6], while exposure to Mn differentially affects GSH in brain regions [7].

On one part, smoking is associated with increased plasma homocysteine levels [8], a precursor of GSH and a tripeptide known as reduced glutathione, which is also a ubiquitous reducing agent whose absence induces severe oxidative stress $(\mathrm{OS})$. It has been suggested that GSH interacts with nitric oxide (NO) by giving S-nitrosoglutathione (GNSO), in the presence of endogenously produced oxygen [9]. GSH is the main redox equilibrium regulator. It plays an important role in the protection of tissues suffering from damage by oxidative agents.

Recent studies indicated that the use of $\mathrm{Zn}$ induces defensive mechanisms in the brain by diminishing free radical-induced lipid peroxidation [10]. Free radicals are reactive oxygen or nitrogen species with impaired electrons, which may induce oxidative damage to biologically important molecules. Although membrane lipids are the main target [11], the central nervous system (CNS) is particularly susceptible to this type of damage, because membrane lipids are known to strongly interact with the lipid bilayer structural proteins [12]. It is necessary to determine the effects of nicotine and oligoelements, because the coexistence of nicotine and micronutrients' deficiency complicates the health of young people. For this, we aim at determining the effect of these substances on dopamine levels and some biomarkers of oxidative stress in juvenile rat brain regions.

\section{Methods}

Fifty six male Wistar rats each with a weight of $80 \mathrm{~g}$ were used in the study. They were divided in single dose and repeated doses, into four experimental groups for each dose and treated as follows: Group $1(\mathrm{n}=7)$, control $(\mathrm{NaCl} 0.9 \%)$; group $2(\mathrm{n}=7)$, nicotine $(1 \mathrm{mg} / \mathrm{rat})$; group 3 $(\mathrm{n}=7)$, oligoelements $50 \mu \mathrm{l} / \mathrm{mg} / \mathrm{kg}$ b.w; and group $4(\mathrm{n}=$ 7), nicotine $(1 \mathrm{mg} / \mathrm{rat})+$ oligoelements $50 \mu \mathrm{l} / \mathrm{mg} / \mathrm{kg}$ b.w. All treatments were administered intraperitoneally every 24 hours for 4 days. Every $100 \mathrm{~mL}$ of oligoelemental solution contains Zinc $55 \mathrm{mg}$, copper $16.90 \mathrm{mg}$, manganese $38.10 \mathrm{mg}$, sodium $163.9 \mathrm{mg}$, fluoride $14 \mathrm{mg}$, iodide $1.3 \mathrm{mg}$, and chloride $25.6 \mathrm{mg}$. The animals were sacrificed by decapitation $30 \mathrm{~min}$ after receiving the last dose of nicotine and oligoelements, and fresh blood was withdrawn to measure their glucose levels. The brains were extracted and stored in $\mathrm{NaCl} 0.9 \%$ at $4{ }^{\circ} \mathrm{C}$. Brain sections obtained from cortex, hemispheres, and cerebellum/medulla oblongata were each homogenized in 5 volumes of TRIS-HCl $0.05 \mathrm{M}, \mathrm{pH} 7.4$ for assessment of lipid peroxidation (TBARS). An aliquot of this tissue was mixed (50:50) with perchloric acid $\left(\mathrm{HClO}_{4}\right) 0.1 \mathrm{M}$, to evaluate the levels of Glutathione (GSH) and dopamine. All experimental procedures were done with authorization of the Laboratory Animals Use and Care Committee of our institution.

The treatments given to animals were as follow:

\begin{tabular}{lcc}
\hline \multicolumn{1}{c}{ Groups } & Single dose & Repeated doses \\
\hline Control $(\mathrm{NaCl} 0.9 \%)$ & $(7)$ & $(7)$ \\
Nicotine $(1 \mathrm{mg} / \mathrm{rat})$ & $(7)$ & $(7)$ \\
Oligoelements $(50 \mu \mathrm{l} / \mathrm{mg} / \mathrm{kg})$ & (7) & (7) \\
$\begin{array}{l}\text { Nicotine }(1 \mathrm{mg} / \mathrm{rat})+\text { oligoelements } \\
(50 \mu \mathrm{l} / \mathrm{mg} / \mathrm{kg})\end{array}$ & (7) & (7) \\
\hline
\end{tabular}

( ) number of animals.

\subsection{Measurement of Blood Glucose}

The procedure to measure glucose in blood was followed in all the animals at the moment of their sacrifice. $20 \mu \mathrm{l}$ of non-anticoagulant fresh blood was placed in a reactive paper of Accu-Chek active equipment (Roche Mannheim Germany). The concentration in $\mathrm{mg} / \mathrm{dl}$ was obtained.

\subsection{Measurement of Glutathione (GSH) in Brain}

The levels of GSH were measured from a sample of the homogenized tissue in $\mathrm{HClO}_{4}$ which was obtained after being centrifuged at $9000 \mathrm{rpm}$ for $5 \mathrm{~min}$. (in a microcentrifuge Mikro 12 - 42, Germany), using the technique reported by Hissin and Hilf [13]. $1.8 \mathrm{ml}$ of Phosphate Buffer at $\mathrm{pH} 8.0$ with EDTA at $0.2 \%$, an aliquot of $20 \mu \mathrm{l}$ of the homogenized tissue in $\mathrm{HClO}_{4}$, and $100 \mu \mathrm{l}$ of ortho-phtaldialdehyde (OPT) in concentration of $1 \mathrm{mg} / \mathrm{ml}$ in methanol, were put in an assay tube and incubated for 15 min. at ambient temperature in total darkness. At the end of incubation, the samples were read in a PERLIN ELMER LS 55 spectrofluorometer with excitation wavelength of $350 \mathrm{~mm}$ and emission of $420 \mathrm{~nm}$. FL Win Lab version 4.00 .02 software was used. The values were inferred in a previously standardized standard curve and were reported in $\mathrm{nM} / \mathrm{g}$ of wet tissue.

\subsection{Measurement of Lipid Peroxidation (TBARS) in Brain}

TBARS determination was carried out based on the technique reported by Gutteridge and Halliwell [14], as described below: from the homogenized brain in tris- $\mathrm{HCl}$ $0.05 \mathrm{M} \mathrm{pH} 7.4,1 \mathrm{ml}$ was taken and to it was added $2 \mathrm{ml}$ of thiobarbaturic acid (Tba) containing $1.25 \mathrm{~g}$ of Tba, 40 $\mathrm{g}$ of trichloroacetic acid (TCA), and $6.25 \mathrm{ml}$ of concentrated hydrochloric acid (HCL) diluted in $250 \mathrm{ml}$ of deionized $\mathrm{H}_{2} \mathrm{O}$. They were heated to boiling point for 30 min. (Thermomix 1420). The samples were then subjected to an ice bath for $5 \mathrm{~min}$ and were centrifuged at $700 \mathrm{~g}$ for $15 \mathrm{~min}$. (Sorvall RC-5B Dupont). The absorb- 
ances of the homogenized tissue were read in triplicate at $532 \mathrm{~nm}$ in a spectrophotometer (Heios-de UNICAM). The concentration of thiobarbituric acid reactive substances (TBARS) was expressed in $\mu \mathrm{M}$ of malondialdehyde/g of wet tissue.

\subsection{Measurement of Dopamine (DA) in Brain}

DA levels were measured in the supernatant of tissue homogenized in $\mathrm{HClO}_{4}$ after centrifugation at $9000 \mathrm{rpm}$ for $10 \mathrm{~min}$ in a microcentrifuge (Hettich Zentrifugen, model Mikro 12 - 42, Germany), with a version of the technique reported by Calderon et al. [15]. An aliquot of the $\mathrm{HClO}_{4}$ supernatant, and $1.9 \mathrm{ml}$ of buffer $(0.003 \mathrm{M}$ octyl-sulphate, $0.035 \mathrm{M} \mathrm{KH}_{2} \mathrm{PO}_{4}, 0.03 \mathrm{M}$ citric acid, $0.001 \mathrm{M}$ ascorbic acid), were placed in a test tube. The mixture was incubated for $5 \mathrm{~min}$ at room temperature in total darkness, and subsequently, the samples were read in a spectrofluorometer (Perkin Elmer LS 55, England) with $282 \mathrm{~nm}$ excitation and $315 \mathrm{~nm}$ emission lengths. The FL Win Lab version 4.00.02 software was used. Values were inferred in a previously standardized curve and reported as nMoles/g of wet tissue.

\subsection{Analysis of Results}

Analysis of variance (two-way ANOVA) with their respective control contrasts after being subjected to variances homogeneity test were used. The values of $p<$ 0.05 were considered statistically significant [16]. To carry out the tests, JMP Statistical Discovery Software version 6.0.0 from SAS was used.

\section{Results}

Table 1 shows the levels of glucose in blood of young rats treated with nicotine in the presences of oligoele-
Table 1. Glucose levels in blood of young rats treated with nicotine and oligoelements.

\begin{tabular}{ccccc}
\hline \multirow{2}{*}{$\begin{array}{c}\text { Schedule } \\
\text { Repeated doses }\end{array}$} & & Groups & Mean & SD \\
& A & Control & 136.71 & 17.62 \\
& B & Nicotine & 163.25 & 19.53 \\
& C & Oligo + Nico & 155.0 & 17.78 \\
& D & Oligoelements & 122.71 & 20.48 \\
Single doses & E & Control & 134.17 & 7.08 \\
& F & Nicotine & 140.67 & 13.99 \\
& G & Oligo + Nico & $155.5^{*}$ & 15.33 \\
& H & Oligoelements & $124.0^{*}$ & 16.53 \\
\hline
\end{tabular}

ANOVA two ways ${ }^{*} \mathrm{p}=0.001$. Oligo + Nico $=$ Oligoelements + Nicotine. $\mathrm{E}$ $\neq \mathrm{G}, \mathrm{G} \neq \mathrm{H}$.

ments. There was a significant increase in this parameter $(\mathrm{p}<0.05)$ in the groups that were treated with oligoelements + nicotine in single dose and significant decrease $(p<0.05)$ in the groups treated with only oligoelements, both with respect to the control group. The concentration of GSH in brain regions of rats treated with nicotine in the presence of oligoelements changed significantly ( $\mathrm{p}=$ 0.001) in different dose treatment (Figure 1). In other words, there were differences between cerebellum/medulla oblongata and hemispheres of the groups treated with single dose, with respect to those treated with repeated doses.

The concentration of lipid peroxidation in the brain regions of rats treated with nicotine and oligoelements (Figure 2), showed significant changes in cerebellum/ medulla oblongata regions in all the experimental groups

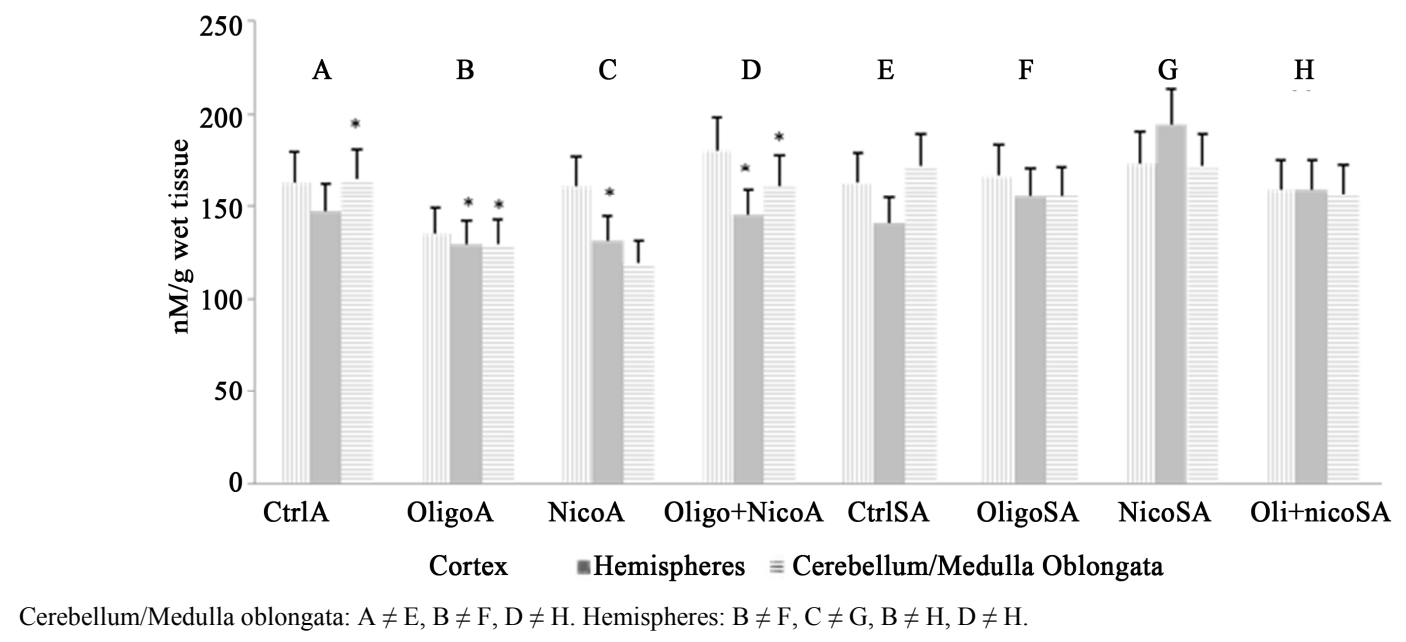

Figure 1. Glutathione (GSH) levels in brain regions of young rats treated with nicotine and oligoelements. ANOVA two ways. ${ }^{*} \mathbf{p}=\mathbf{0 . 0 0 1}$, Oligo + Nico $=$ Oligoelements + Nicotine. 


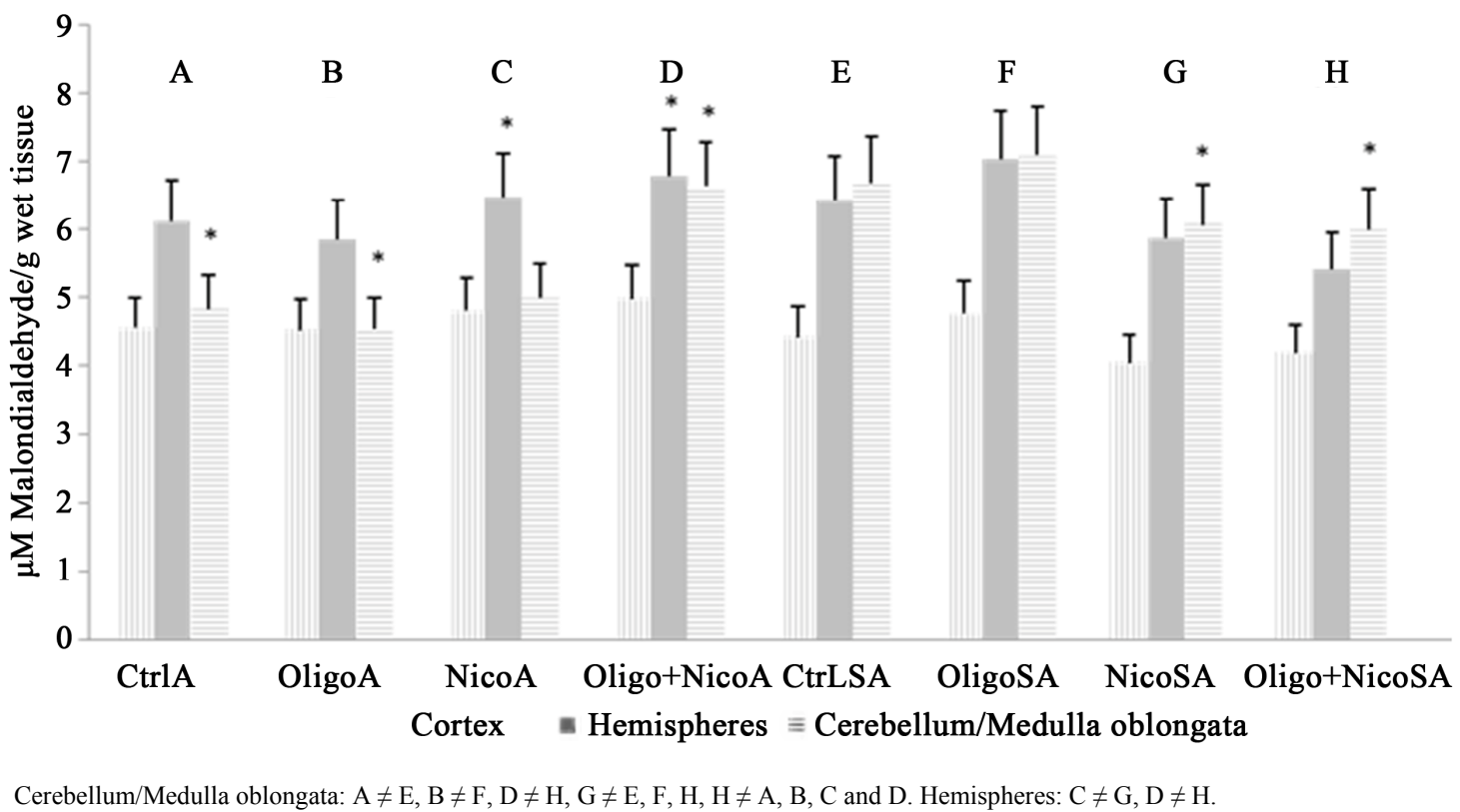

Figure 2. Lipid peroxidation levels in brain regions of young rats treated with nicotine and oligoelements. ANOVA two ways. ${ }^{*} \mathrm{p}=\mathbf{0 . 0 0 1}$, Oligo + Nico $=$ Oligoelements + Nicotine.

with single dose when compared with those treated with repeated doses. In the region of hemisphere of rats that received just oligoelements, lipoperoxidation levels significantly decreased $(p<0.05)$, and increased in the groups treated with single dose of oligoelements + nicotine when compared with those treated with repeated doses.

Figure 3 shows the levels of dopamine in the brain regions of the rats treated with nicotine and oligoelements. All experimental groups with single dose showed higher dopamine levels in cerebellum/medulla oblongata than those with repeated doses. This effect was particularly notable if nicotine group was compared with oligoelements + nicotine using two-way ANOVA analysis with the later showing significant differences $(p<0.05)$, regardless of the treatment received. In cortex, this indicator showed a higher increase in all experimental groups with single dose than in those with repeated doses.

Dopamine levels in hemispheres of oligoelements + nicotine group treated with single dose significantly decreased when compared with repeated doses (two-way ANOVA, $p<0.05$ ). Likewise, this control group showed higher levels of dopamine $(p<0.05)$, compared with control group of repeated doses.

\section{Discussion}

Nicotine replacement therapy is a safer and a more controlled substitute for smoking than ST [17]. In any case, these therapies are made for adults and completely keep aside the adolescent smokers. Based on this, we proposed in the present work the evaluation of nicotine effects in adolescent animal models.

The levels of glucose increased in groups of animals treated with nicotine, because this substance induces increases in the glycolytic pathway and Krebs cycle of neurons, as well as in brain blood flow [18].

The levels of GSH reduced in cerebellum/medulla oblongata and hemisphere regions principally by the administration of a single dose of nicotine. This situation was found also with the oligoelements. This effect on GSH coincides with the reports of Sreekala and Indira [19], who suggest that nicotine alone increases lipoperoxidation by the inhibition of antioxidant enzymes and when combined with oligoelements like selenium, the effect is inverted. Also, the decrease could be due to the interaction with NO [9], which was produced by the inhibition of mitochondrial electron transport chain (MitoETC) complexes (I, II, III), in different brain regions [20]. Particularly GSH measurement in medulla oblongata is very different from the other regions because it was more susceptible to oxidative stress as some studies suggested [21].

Lipoperoxidation increased in the cerebellum/Medulla oblongata and hemisphere regions in co-administration of single dose of nicotine and oligoelements as well as in nicotine alone. With the use of only oligoelements, this effect decreased in these regions which did not agree with the findings of Carmona et al, [22], who suggest that nicotine attenuates the formation of peroxides, and might reduce the production of beta-amyloid proteins in Alzheimer's disease with chronic treatment doses, and may be a novel protective therapy in Alzheimer's disease [23]. With respect to the region of the cortex, there was 


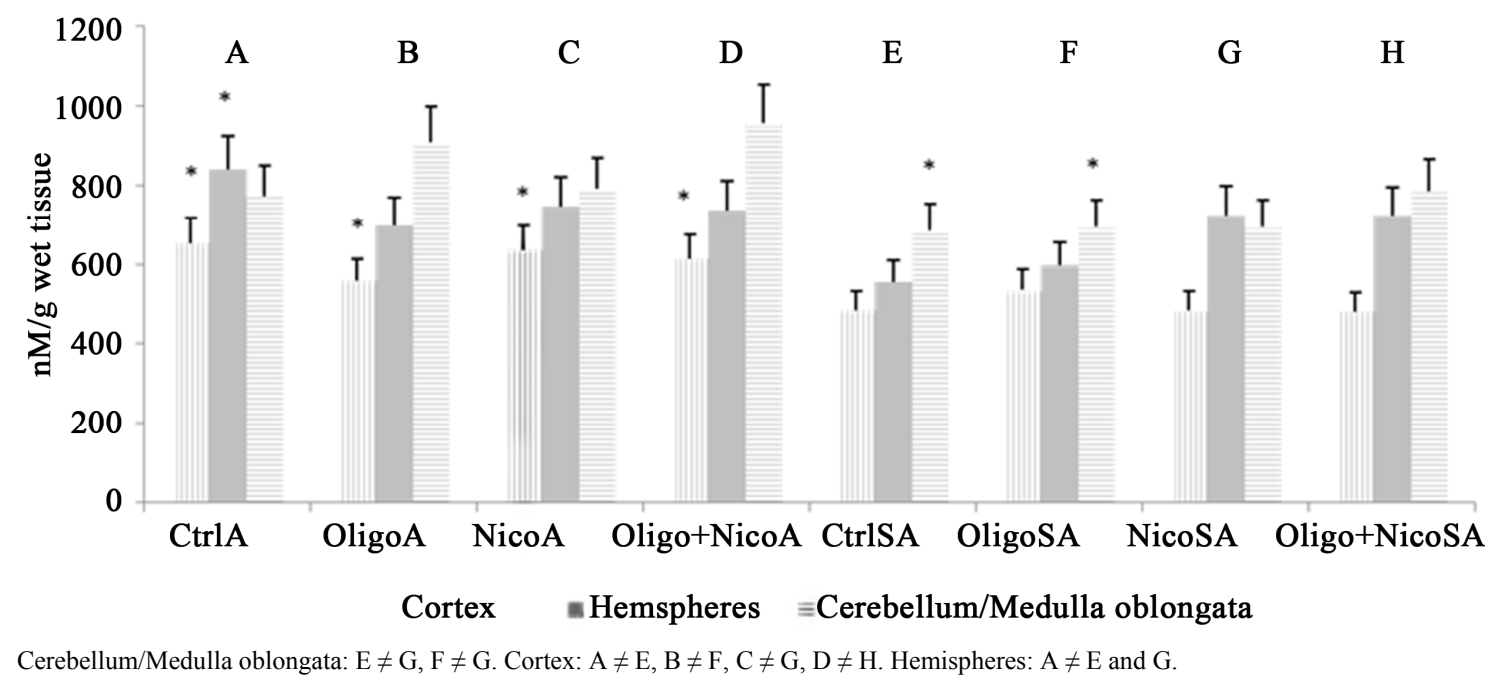

Figure 3. Dopamine levels in brain regions of young rats treated with nicotine and oligoelements. ANOVA two ways. ${ }^{*} \mathrm{p}=$ 0.0001, Oligo + Nico $=$ Oligoelements + Nicotine.

no finding of any effect to the treatment in all the animals, although recent studies suggest that the presence of nicotine increases the formation of peroxides in this region [24].

In general, dopamine levels decreased in cortex and hemisphere regions with co-administration of nicotine and oligoelements in unique doses. On the other hand, this effect was not found in cerebellum/medulla oblongata where there was an increase of dopamine by the combined administration of the two substances in the same doses. This effect was in accordance with the findings of Tariq et al. [25], who suggested that inhibition of nicotine on decreasing DA, which is induced by 3-nitropropionic acid, is due thanks to nicotinic acetylcholine receptors (nAChRs). These receptors could be responsible for the absence of significant statistical difference in the indicators evaluated in this study with chronic dosis of nicotine, probably due to the modulation of these receptors by oligoelements like zinc and the concentration, and time of exposure to nicotine, suggesting that zinc potency depends on the degree of desensitization [26].

In the present study developed with adolescent animal model and based on the results of some studies, the mechanism proposed for the inhibition of tobacco (nicotine) consumption in adolescent subjects is to reduce sugar-rich food consumption and substitute it with oligoelement-rich food [5], because if this source of sugar is reduced, dopamine (DA) systems are activated and lesions of dopaminergic neurons or pharmacological blockade of DA receptors seem to reduce the reward value of both sweet tastes and drugs of abuse [27]. Considering the above results, we conclude that nicotine and oligoelements are associated with increase in glucose levels, an effect which was more pronounced in the group of animals treated with single dose of both drugs, probably by no inhibition of fatty acid oxidation on brain regions [28], because glucose determine fatty acid oxidation by controlling the rate of long-chain fatty acid entrance into the mitochondria [29]. Reduction of oxidative stress and dopamine metabolism may be involved in these effects.

\section{Conclusion}

Nicotine and oligoelements are associated with increase in glucose levels on blood. It induces reduction of oxidative stress and dopamine metabolism on brain regions.

\section{Acknowledgements}

We thank Dr. Cyril Ndidi Nwoye, who helps us to translate the manuscript.

\section{REFERENCES}

[1] ENA, "National Survey of Addictions," SSA, Conadic, INP, DGE, INSP, 2008.

[2] C. Kalpana, A. R. Sudheer, K. N. Rajasekharan and V. P. Menon, "Comparative Effects of Curcumin and Its Synthetic Analogue on Tissue Lipid Peroxidation and Antioxidant Status during Nicotine-Induced Toxicity," Singapore Medical Journal, Vol. 48, No. 2, 2007, pp. 124-130.

[3] R. Chatkin and J. M. Chatkin, "Smoking and Changes in Body Weight: Can Physiopathology and Genetics Explain This Association?" Journal of Brasileiro de Pneumologia, Vol. 33, No. 6, 2007, pp. 712-719.

[4] M. Flores, N. Macías, M. Rivera, S. Barquera, L. Hernández, G. A. García and J. Rivera, "Energy and Nutrient Intake among Mexican School-Aged Children, Mexican National Health and Nutrition Survey 2006," Salud Publica de Mexico, Vol. 51, No. 4, 2009, pp. S540-S550.

[5] G. D. Calderón, G. E. Hernández, M. G. Barragán and A. 
L. Carmona, "Pediatric Trace Elements. Clinical Updates," Acta Pediatrica de Mexico, Vol. 32, 2011, pp. 287-291.

[6] D. Ozcelik and H. Uzun, "Copper Intoxication; Antioxidant Defenses and Oxidative Damage in Rat Brain," Biological Trace Element Research, Vol. 127, No. 1, 2009, pp. 45-52. doi:10.1007/s12011-008-8219-3

[7] K. M. Erikson, D. C. Dorman, L. H. Lash and M. Aschner, "Manganese Inhalation by Rhesus Monkeys Is Associated with Brain Regional Changes in Biomarkers of Neurotoxicity," Toxicology Science, Vol. 97, No. 2, 2007, pp. 459-466. doi:10.1093/toxsci/kfm044

[8] D. B. Kim, Y. S. Oh, K. D. Yoo, J. N. Lee, C. S. Park, S. H. Ihm, S. W. Jang, B. J. Shim, H. Y. Kim, K. B. Seung, T. H. Rho and J. H. Kim, "Passive Smoking in NeverSmokers Is Associated with Increased Plasma Homocysteine Levels," International Heart Journal, Vol. 51, No. 3, 2010, pp. 183-187. doi:10.1536/ihj.51.183

[9] M. Benuck, S. M. Banay, T. De Guzman and A. Lajtha, "Effect of Food Deprivation on Glutathione and Amino Acid Levels in Brain and Liver of Young and Aged Rats," Brain Research, Vol. 678, No. 1-2, 1995, pp. 259264. doi:10.1016/0006-8993(95)00204-4

[10] C. S. Bediz, A. K. Baltaci, R. Mogulkoc and E. Oztekin, "Zinc Supplementation Ameliorates Electromagnetic FieldInduced Lipid Peroxidation in the Rat Brain," Tohoku Journal of Experimental Medicine, Vol. 208, No. 2, 2006, pp. 133-140.

[11] J. S. Beckman, T. W. Beckman, J. Chen, P. A. Marshall and B. A. Freeman, "Apparent Hydroxyl Radical Production by Peroxynitrite: Implications for Endothelial Injury from Nitric Oxide and Superoxides," Proceeding $\mathrm{Na}$ tional of Academic Science USA, Vol. 87, No. 4, 1990, pp. 1624-1629.

[12] I. Swapna, K. V. Sathya and C. R. Murthy, "Membrane Alterations and Fluidity Changes in Cerebral Cortex during Ammonia Intoxication," Neurology and Toxicology, Vol. 335, 2005, pp. 700-704.

[13] P. J. Hissin and R. Hilf, "A Flurometric Method for Determination of Oxidized and Reduced Glutathione in Tissue," Analitical Biochemistry, Vol. 74, No. 1, 1976, pp. 214-226. doi:10.1016/0003-2697(76)90326-2

[14] J. M. Gutteridge and B. Halliwell, "The Measurement and Mechanism of Lipid Peroxidation in Biological Systems," Trends in Biochemical Sciences, Vol. 15, No. 4, 1990, pp. 129-135. doi:10.1016/0968-0004(90)90206-Q

[15] G. D. Calderón, B. N. Osnaya, A. R. García, G. E. Hernández, P. A. Guillé and O. H. Juárez, "Levels of Glutathione and Some Biogenic Amines in the Human Brain Putamen after Traumatic Death," Proceeding of Western Pharmacology Society, Vol. 51, 2008, pp. 25-29.

[16] L. Castilla-Serna and J. Cravito, "Estadística Simplificada Para la Investigación en Ciencias de la Salud," Editorial Trillas, México City, 1991.

[17] Z. Arabi, "Metabolic and Cardiovascular Effects of Smokeless Tobacco," Journal of the Cardiometabolic Syndrome, Vol. 1, No. 5, 2006, pp. 345-350. doi:10.1111/j.1559-4564.2006.05930.x

[18] A. Toledano, M. I. Alvarez and D. A. Toledano, "Diversity and Variability of the Effects of Nicotine on Different
Cortical Regions of the Brain: Therapeutic and Toxicological Implications," Central Nervous System Agents in Medicinal Chemistry, Vol. 10, No. 3, 2010, pp. 180-206. doi:10.2174/1871524911006030180

[19] S. Sreekala and M. Indira, "Effects of Exogenous Selenium on Nicotine-Induced Oxidative Stress in Rats," Biological Trace Element Research, Vol. 130, No. 1, 2009, pp. 62-71. doi:10.1007/s12011-009-8314-0

[20] S. Das, N. Gautam, S. K. Dey, T. Maiti and S. Roy, “Oxidative Stress in the Brain of Nicotine-Induced Toxicity: Protective Role of Andrographis Paniculata Nees and Vitamin E," Applied Physiology Nutrition and Metabolism, Vol. 34, No. 2, 2009, pp. 124-135. doi:10.1139/H08-147

[21] K. V. Sathyasaikumar, I. Swapna, P. V. Reddy, C. R. Murthy, G. A. Dutta, B. Senthilkumaran and P. Reddanna, "Fulminant Hepatic Failure in Rats Induces Oxidative Stress Differentially in Cerebral Cortex, Cerebellum and Pons Medulla," Neurochemical Research, Vol. 32, No. 3, 2007, pp. 517-524. doi:10.1007/s11064-006-9265-x

[22] P. Carmona, C. A. Rodríguez, I. Alvarez, E. deMiguel and A. Toledano, "FTIR Microspectroscopic Analysis of the Effects of Certain Drugs on Oxidative Stress and Brain Protein Structure," Biopolymers, Vol. 89, No.6, 2008, pp. 548-554. doi:10.1002/bip.20944

[23] A. Nordberg, L. E. Hellström, M. Lee, M. Johnson, M. Mousavi, R. Hall, E. Perry, I. Bednar and J. Court, "Chronic Nicotine Treatment Reduces Beta-Amyloidosis in the Brain of a Mouse Model of Alzheimer's Disease (APPsw)," Journal of Neurochemistry, Vol. 81, No. 3, 2002, pp. 655-658. doi:10.1046/j.1471-4159.2002.00874.x

[24] D. M. Arrick and W. G. Mayhan, "Acute Infusion of Nicotine Impairs nNOS-Dependent Reactivity of Cerebral Arterioles via an Increase in Oxidative Stress," Journal of Applied Physiology, Vol. 103, No. 6, 2007, pp. 20622067. doi:10.1152/japplphysiol.00411.2007

[25] M. Tariq, H. A. Khan, I. Elfaki, S. Al Deeb and K. Al Moutaery, "Neuroprotective Effect of Nicotine against 3-Nitropropionic Acid (3-NP)-Induced Experimental Huntington's Disease in Rats," Brain Research Bulletin, Vol. 67, No. 1-2, 2005, pp. 161-168. doi:10.1016/i.brainresbull.2005.06.024

[26] G. E. Vázquez and C. J. García, "Neuronal Nicotinic Acetylcholine Receptors Are Modulated by Zinc," Neuropharmacology, Vol. 56, No. 6-7, 2009, pp. 1035-1040. doi:10.1016/j.neuropharm.2009.02.008

[27] C. M. Cannon and M. R. Bseikri, "Is Dopamine Required for Natural Reward?" Physiology Behaviour, Vol. 81, 2004, pp. 741-748. doi:10.1016/j.physbeh.2004.04.020

[28] T. K. Makar, B. L. Hungund, G. A. Cook, K. Kashfi and A. J. Cooper, "Lipid Metabolism and Membrane Composition Are Altered in the Brains of Type II Diabetic Mice," Journal of Neurochemistry, Vol. 64, No. 5, 1995, pp. 2159-2168. doi:10.1046/j.1471-4159.1995.64052159.x

[29] L. S. Sidossis, C. A. Stuart, G. I. Shulman, G. D. Lopaschuk and R. R. Wolfe, "Glucose Plus Insulin Regulate Fat Oxidation by Controlling the Rate of Fatty Acid Entry into the Mitochondria," Journal of Clinical Investigation, Vol. 98, No. 10, 1996, pp. 2244-2250. doi:10.1172/JCI119034 